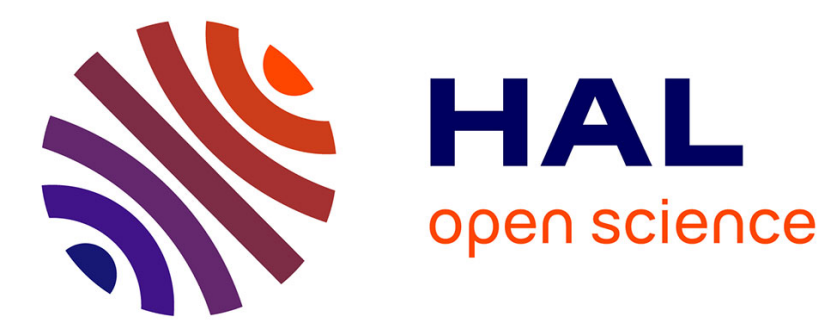

\title{
Mieux comprendre les cyberconsommateurs pour accroître les achats en ligne
}

\author{
Nathalie Audigier
}

\section{To cite this version:}

Nathalie Audigier. Mieux comprendre les cyberconsommateurs pour accroître les achats en ligne.

Gestion - HEC Montréal, 2009, 34, pp.78 - 78. 10.3917/riges.344.0078 . hal-01570895

\section{HAL Id: hal-01570895 \\ https://hal.science/hal-01570895}

Submitted on 1 Aug 2017

HAL is a multi-disciplinary open access archive for the deposit and dissemination of scientific research documents, whether they are published or not. The documents may come from teaching and research institutions in France or abroad, or from public or private research centers.
L'archive ouverte pluridisciplinaire HAL, est destinée au dépôt et à la diffusion de documents scientifiques de niveau recherche, publiés ou non, émanant des établissements d'enseignement et de recherche français ou étrangers, des laboratoires publics ou privés. 
Mieux comprendre les cyberconsommateurs pour accroître les achats en ligne

\section{Nathalie Audigier}

Nathalie Audigier est maître de conférences en gestion à l'Université Antilles-Guyane (France), nathalie.audigier@wanadoo.fr. 


\section{Résumé}

L'achat en ligne donne souvent des résultats décevants. Ainsi, plusieurs facteurs expliquent les limites du marketing en ligne : l'absence de réactivité des sites, qui ne sont souvent que des catalogues en ligne ou des vitrines virtuelles; l'inadéquation de l'outil au besoin immédiat, de sorte que les bannières ne génèrent pas les achats ou les demandes d'informations attendus; enfin, l'inadaptation des sites aux véritables comportements d'achat, l'achat en ligne n'étant pas approprié pour tous les produits et services. Afin de rendre les outils en ligne réactifs et adaptés aux comportements d'achat, les spécialistes du marketing doivent mieux appréhender les comportements des internautes, en recourant à divers outils (entrevues, suivi informatique, sondages, etc.). Ils devraient également prendre les moyens suivants : s'assurer que le site Web tient compte du processus d'achat en entier, puis concevoir un système ouvert intégrant les processus d'achat en ligne et physique. L'article se termine par une discussion à propos des bénéfices liés à l'amélioration du marketing en ligne.

Mots clés : cyberconsommateurs, achat en ligne, processus d'achat, marketing en ligne, comportements d'achat 


\section{Mieux comprendre les cyberconsommateurs pour accroître les achats en ligne Nathalie Audigier}

Une récente enquête (Benchmark Group, 2009) indique que $65 \%$ des achats sur Internet ne sont pas menés à leur terme. Si certains internautes changent tout simplement d'avis en cours de route, d'autres s'égarent sur les sites. Une autre étude, menée en 2002, par Monster et al., montre qu'une forte majorité d'internautes interrompent leur magasinage faute de trouver l'information recherchée.

\section{Quels défis le marketing en ligne doit-il relever?}

Devant cette situation, l'absence de réactivité des sites est très souvent mise en cause. En effet, dans bien des cas, les sites Web ne sont que des catalogues en ligne ou des vitrines virtuelles. Ils passent totalement à côté de la formidable capacité d'Internet à se transformer en un outil de réponse aux besoins précis du client à un moment donné. Si, à peine connecté au site, l'internaute lance une recherche par mots clés, c'est qu'il souhaite acheter un produit particulier, et non feuilleter un catalogue. À partir de cette information circonstancielle, les sites devraient pouvoir adapter la suite des écrans.

L'échec relatif du marketing en ligne s'explique également par l'inadéquation de l'outil au besoin immédiat. Ainsi, on note que les bannières ne génèrent pas les achats ou les demandes d'informations attendus (les internautes ne cliquent pas dessus). Pourtant, une étude du Benchmark Group (2007) révèle qu'elles sont très efficaces pour ce qui est de la notoriété de la marque, puisque $50 \%$ des visiteurs remarquent la bannière et $11 \%$ s'en souviennent 24 heures plus tard (référence).

Par ailleurs, les outils sont peu souvent adaptés aux véritables comportements d'achat. Ainsi, il est illusoire de se fier outre mesure aux communautés en ligne pour influencer les clients. Certains produits sont trop complexes et trop chers pour que le client prenne sa décision sans consulter un spécialiste ou se rendre au magasin pour toucher le produit, voire l'essayer. Une fois encore, le temps et l'expérience 
prouvent que les outils en ligne, comme leurs homologues physiques, doivent s'adapter aux comportements d'achat réels.

\section{Le besoin de mieux comprendre les comportements d'achat en ligne}

Afin de rendre les outils en ligne réactifs et adaptés au comportement d'achat, les spécialistes du marketing doivent tout d'abord appréhender les comportements des internautes face à leurs produits et services. Généraliser à plusieurs catégories d'offres les résultats d'une enquête sur les comportements en ligne est une grossière erreur, mais malheureusement courante. Si personne ne conteste le fait qu'il existe une différence entre l'achat d'une voiture chez un concessionnaire et l'achat d'un tube de dentifrice dans un supermarché, personne n'opère la même distinction en ce qui concerne les achats en ligne.

Les responsables du marketing doivent également être conscients que les comportements changent selon les circonstances. Ainsi, l'internaute qui veut aujourd'hui prendre le temps de réfléchir pour s'assurer de faire le bon choix pourra souhaiter demain procéder rapidement parce qu'il manque de temps. Par ailleurs, il est essentiel de prendre en considération le caractère souvent anxiogène que revêt l'achat en ligne (peur de se tromper dans les modalités de sélection des produits, au cours du processus de paiement, etc.), notamment pour les cyberacheteurs néophytes.

Les comportements d'achat sont, de façon globale, très complexes, mais de nombreux chercheurs, qui appuient leur réflexion sur le marketing traditionnel, essaient de modéliser ces mécanismes et s'attachent à en faciliter la compréhension en différenciant chacune de leurs étapes. On peut, par exemple, distinguer les processus en se fondant sur le niveau d'implication du client dans l'acte d'achat (qui augmente proportionnellement au prix du produit) et sur le degré de différenciation des offres. Toutefois, on remarque que le niveau d'implication du client n'est pas identique à l'intérieur d'une même catégorie de produits. Certains articles peuvent susciter un achat d'impulsion (un vêtement de sport), tandis que d'autres donneront lieu à un achat mûrement réfléchi (une tenue de soirée). De même, le degré de 
différenciation perçu des produits n'est pas fixe non plus : en la matière, le client est le seul juge. C'est sa perception, et non un critère objectif, qui définit ce produit comme un produit banal ou non. Cela implique que le degré d'originalité du produit évolue avec la perception du client, qui est elle-même influencée par des facteurs tels que les campagnes publicitaires... ou son humeur.

Ces deux axes - implication du client et différenciation des offres - ont des conséquences multiples sur la façon dont les clients s'informent et se forgent une opinion sur le produit et, en définitive, font leurs choix. Ainsi, pour des produits à faible implication, il suffit au client de l'acheter une fois pour l'essayer et s'en faire une idée. Dans le cas de produits à plus forte implication, comme les voyages, le client a déjà une idée précise de la question depuis longtemps, parfois même avant de déterminer ses dates de vacances.

En partant de ces constats, on doit considérer que le comportement du consommateur peut varier tout au long du cycle ou processus d'achat, en raison du plus ou moins grand besoin d'informations associé à chaque étape. Si on décompose le processus en cinq étapes - intention d'achat, intérêt, préférence, achat, fidélité -, on remarque, pour un achat habituel, que l'étape de l'intérêt ne requiert que quelques informations, alors que dans le cas d'un achat complexe, elle suppose des recherches et une évaluation. La conception des sites Web doit donc intégrer cette réalité. Il faut, pour cela, approfondir la segmentation en amont des clients en fonction de leurs comportements.

\section{Comment étudier le cyberconsommateur pour mieux le comprendre?}

On ne peut imaginer qu'une grande enseigne de la distribution implante un supermarché dans lequel plus de la moitié des visiteurs se perdent et repartent les mains vides ou qu'un restaurateur oblige ses clients à faire le tour de chacune des tables avant d'atteindre le panneau sur lequel se trouve son menu. C'est pourtant ce à quoi ressemblent bon nombre de boutiques en ligne. La raison majeure pour laquelle les services en ligne suscitent encore des réticences auprès du public est, d'après de nombreux sondages, 
que ces services ne répondent pas aux attentes des visiteurs. Ils sont en effet confus et les utilisateurs s'y égarent facilement. Le seul moyen de s'assurer de la conformité des sites aux attentes des cyberacheteurs consiste à effectuer divers tests s'appuyant sur diverses approches.

Premièrement, on peut recourir au laboratoire d'«utilisabilité». Il s'agit généralement d'entretiens seul à seul avec des personnes dont le profil est représentatif des utilisateurs du site. L'animateur demande au participant d'accomplir des tâches (par exemple, commander un produit ou trouver une information particulière) et note le résultat. Cette méthode permet d'observer le comportement des visiteurs du site, non seulement en relation avec leurs progrès dans l'exécution d'une tâche (le nombre de clics, le chemin parcouru sur le site, etc.), mais aussi en fonction de leurs réactions émotionnelles hors ligne (grimace de dégoût ou de dépit, énervement face à la complexité de la tâche, etc.). Grâce à un tel test, on peut découvrir que certains clients potentiels ont peur de cliquer sur le bouton «Commander», car ils pensent qu'ils sont obligés de passer une commande, que cette sélection sera irréversible ou que leur compte sera débité automatiquement.

Deuxièmement, ce type de tests peut aussi être conduit entièrement en ligne, dans le cas où les visiteurs sont issus de différents pays. Le principe est alors de mettre en place un outil de pistage (tracking) de chaque mouvement sur le site, pour suivre exactement les réactions des visiteurs, le temps de lecture de chaque écran, le taux d'abandon, etc. Le seul problème des tests en ligne est qu'ils ne permettent pas d'enregistrer totalement les réactions émotionnelles (langage corporel), sauf dans le cas où il est possible de disposer d'une webcaméra en complément. En revanche, ces tests sont particulièrement instructifs en ce qui concerne la prise en compte de l'environnement de l'internaute (lieu de la consultation en ligne, moment de la journée, urgence de la démarche, etc.), des facteurs externes (bruits, dérangements divers, etc.) et de la bande passante. 
Troisièmement, une autre possibilité consiste à placer un mini-sondage (posant la question «Que pensez-vous de cette page?» ou «Quel est votre degré de satisfaction au regard de cet écran?») sur chacune des pages d'un site et de recouper ces informations avec les fichiers journaux pour en déduire la qualité de l'expérience de l'utilisateur. L'utilisation conjointe de logiciels d'analyse en ligne (Webalizer ou Speed-Trap, par exemple) permet d'affiner les connaissances sur les internautes en proposant des données quantitatives et qualitatives relatives à leur navigation.

Finalement, une autre option est de créer une série de tâches, comme pour un test en laboratoire, et de mesurer le degré de réalisation de chaque objectif.

\section{Comment rendre le marketing en ligne plus performant?}

L'entreprise doit donc évaluer le degré d'adéquation de son site aux comportements des clients. Il s'agit pour celle-ci de s'assurer que le site répond aux besoins des clients compte tenu de leur processus d'achat probable, qu'il prévoit les comportements atypiques éventuels - comme l'achat d'impulsion d'un équipement coûteux - et qu'il fournit un soutien tout au long du processus de décision, de l'intention d'achat à la fidélisation. Par ailleurs, compte tenu de l'évolution du paysage commercial, les responsables du marketing doivent songer à ériger des passerelles entre les différents canaux de distribution et considérer de façon globale le nouvel acheteur en intégrant la démarche virtuelle et la démarche physique de son processus d'achat. Nous développons ci-après ces deux voies d'amélioration du marketing en ligne.

\section{S'assurer que le site Web tient compte du processus d'achat en entier}

Adapter un site Web aux comportements des clients implique d'élargir le concept du site pour intégrer l'ensemble de la présence marketing en ligne de l'entreprise. Cette nouvelle approche recouvre tous les moyens utilisés par Internet pour engager avec les clients un dialogue destiné à mieux cerner leurs besoins, notamment les nouvelles techniques de marketing en ligne : citons, par exemple, les listes d'inclusion de courriels (opt-in mails), où des courriels sont envoyés aux destinataires seulement avec leur accord, et le marketing viral. 
Pour les spécialistes, la principale difficulté consiste aujourd'hui à choisir le meilleur outil de marketing en ligne, celui qui mérite l'investissement. Beaucoup estiment que pour mettre toutes les chances de son côté, il faut utiliser tous ces outils - une conception que la presse professionnelle tend à renforcer. Cependant, chaque outil de marketing en ligne correspond à un type précis de comportement à une étape du processus d'achat, de la même façon que les divers supports médiatiques traditionnels permettent d'agir sur différents ressorts comportementaux de l'acheteur. À titre d'exemple, les revendeurs de matériel hi-fi dernier cri, produit complexe s'il en est, doivent miser davantage sur la liste d'inclusion et sur l'événementiel que sur les bannières. Hélas, les entreprises qui évaluent les investissements dans le marketing en ligne de façon aussi systématique sont encore trop rares.

À l'image de ce qui se fait dans les plans médias traditionnels, il est souhaitable de combiner les outils pour accompagner l'ensemble du processus. Ainsi, Amazon.com entretient sa visibilité grâce à des vitrines sur des sites partenaires. Contre le versement d'une redevance modique par vente, le libraire en ligne place des liens sur des sites partenaires ciblés. L'intention d'achat ainsi suscitée est ensuite transformée en intérêt par la présentation d'éditoriaux et de critiques de livres. L'intérêt glisse vers la préférence grâce aux recommandations de livres similaires ou sur le même sujet. Puis, la préférence se transforme en achat grâce à l'omniprésence de l'icône «Ajouter au panier» et «Commander en 1 clic».

Anticiper l'intention du client favorise la progression du processus d'achat. En éliminant certains risques liés à l'achat - notamment le risque financier qui, comme nous l'avons précisé, est souvent ressenti fortement par l'internaute - et en intégrant le processus d'achat en cours, il est possible d'accompagner le client de l'étape de la simple préférence à celle de l'achat mû par un sentiment de confiance. En fait, quelques questions rapides et bien ciblées sur l'écran peuvent permettre au client d'avancer dans le processus d'achat, et à l'entreprise d'améliorer ses capacités de vente incitative (upselling). 
L'idéal reste toutefois de pouvoir anticiper de nombreux comportements simultanément. Certains sites sont conçus pour gérer plusieurs types de comportements grâce à des menus déroulants permettant au client de se placer dans la situation d'achat qui est la sienne. Ainsi, dès lors qu'un internaute choisit une catégorie de produits à partir du menu déroulant, le site lui propose une sélection adaptée à cette circonstance. Chaque proposition offre alors de nouveaux critères de sélection (par prix, par fonctionnalité, etc.), chacun étant suivi d'un lien «Plus dans cette catégorie» qui permet d'affiner la recherche. Pour faciliter - et accélérer - le passage à l'achat, chaque option est assortie d'un bouton «Commander maintenant» qui élimine la page «Ajouter au panier».

Pour créer des sites capables de répondre à plusieurs segments de comportement d'achat, il convient donc d'intégrer les connaissances sur la progression de chaque type de comportement dans le processus d'achat, qui va de l'intention d'achat à l'instauration de la fidélité, comme nous l'avons indiqué. Mais ces techniques ne concernent que le comportement de l'internaute. Cependant, il ne faut pas négliger le fait que ce cyberacheteur est aussi un consommateur physique. Cela implique une intégration parfaite des comportements en ligne et hors ligne.

\section{Concevoir un système ouvert intégrant les processus d'achat en ligne et physique}

Une analyse approfondie du client constitue donc le point de départ essentiel pour améliorer la connaissance de son comportement en ligne et apporter les réponses adéquates. Cependant, une fois connecté, le cyberconsommateur peut changer de comportement, voire se transformer en consommateur physique. Ainsi, les canaux traditionnels (magasins) peuvent être utilisés pour des achats réfléchis ou pour l'obtention d'informations, tandis qu'Internet est susceptible de servir à des achats d'impulsion ou à des achats répétitifs ou encore à une comparaison des offres. Ainsi, il se dessine là une version imbriquée du processus d'achat, dans laquelle le consommateur passe instantanément de la visite d'une boutique virtuelle à la fréquentation d'une galerie marchande. 
Force est de constater qu'Internet ne satisfait pas, aujourd'hui, tous les besoins des consommateurs. Cela explique peut-être l'intérêt suscité par le concept de clic et mortier (brick and click), association du traditionnel et du virtuel. La volonté d'intégrer les deux canaux a donné naissance à des innovations comme la possibilité d'acheter en ligne et de retourner la marchandise dans les boutiques. Mais la coordination n'est pas encore au rendez-vous. Il est nécessaire que les entreprises envisagent la formation de leurs vendeurs à la gestion des retours de produits achetés sur le Web et surtout définissent un processus de correction des défauts (en ligne ou en magasin) par anticipation, sans attendre les sollicitations des clients. La réussite d'une telle organisation passe par la diminution des risques ressentis par l'acheteur (en facilitant la procédure de retour, par exemple), la valorisation de son implication (par une politique de prix attrayants en ligne), la réduction de la différenciation perçue (par la présentation de témoignages et l'offre d'échantillons) et l'enrichissement de l'offre proposée (par un forfait intégrant des services).

Enfin, un système ouvert, offrant de multiples passerelles entre les canaux virtuels et les canaux physiques, s'impose. Ainsi, chez certaines entreprises de vente par correspondance, les utilisateurs ont la possibilité de rechercher en ligne les produits par référence, qu'ils trouvent dans le catalogue envoyé à domicile et qui sont livrés dans les boutiques de l'enseigne. Et rares sont les prospectus, les sacs d'emballage ou les publicités qui ne mentionnent pas une adresse Web. Voilà autant de passerelles entre les deux mondes qui favorisent l'intégration. Mais ces liens ne suffisent pas. Les clients doivent pouvoir utiliser l'outil - en ligne ou non - qui correspond le mieux à leurs besoins. Ainsi, pour les clients qui aiment toucher ou voir le produit avant de se décider, on peut imaginer l'envoi d'échantillons (contre une somme modique pour limiter les frais commerciaux); pour les clients qui ont besoin d'être rassurés sur la livraison des produits achetés sur le Net, on peut proposer le suivi en ligne de la commande. En permettant à chaque canal, en ligne ou non, de donner le meilleur de lui-même, les spécialistes du marketing favorisent l'utilisation du nouveau canal intégré et, au final, stimulent les ventes. 


\section{$\underline{\text { Les bénéfices liés à l'amélioration du marketing en ligne }}$}

Cette approche du comportement du cyberconsommateur crée de nouvelles possibilités stratégiques. Tout d'abord, elle permet aux entreprises d'influer sur le comportement du consommateur tout au long du processus d'achat. Certaines techniques traditionnelles, améliorées par le marketing en ligne, peuvent être utilisées pour agir sur le comportement du consommateur à l'avantage de l'entreprise, comme nous en avons donné quelques exemples : si le produit est lui-même porteur d'atouts probants, l'entreprise doit mettre l'accent sur l'achat complexe; si le produit bénéficie d'une bonne réputation mais comprend une technologie désuète, elle doit se concentrer sur l'étape de la réduction de la dissonance.

Cette approche met également au jour des segments de clientèle laissés pour compte. Si, naturellement, la plupart des entreprises seront tentées de servir les segments les plus directement concernés, les possibilités que comportent les autres segments ne doivent pas être négligées. Ainsi, si des millions de femmes aiment essayer régulièrement de nouveaux cosmétiques, d'autres restent fidèles aux mêmes pendant des années : étant donné que le fait de parier sur leur fidélité éternelle constitue un risque trop important dans la situation que connaît actuellement le secteur, pourquoi ne pas leur proposer un système de réapprovisionnement automatique de leurs produits préférés?

Au final, c'est le client qui détermine la meilleure stratégie du nouveau marketing. Il ne s'agit pas du client défini comme segment de valeur ou démographique, mais de celui défini par les circonstances qui entourent son acte d'achat, le client sous toutes ses formes : le gestionnaire pressé qui va prendre son avion et le même gestionnaire qui feuillette tranquillement le magazine de la compagnie aérienne; la femme au foyer qui remplit son caddie au supermarché accompagnée de ses trois enfants, et la même, qui, le soir, fait du magasinage sur Internet quand les enfants sont couchés. 
Plus maniables, ces segments «circonstanciels» permettent aux entreprises de prendre une longueur d'avance en ciblant les différents comportements grâce à une combinaison efficace des capacités en ligne et des capacités traditionnelles. Mais les entreprises qui seront dans le peloton de tête seront celles qui utiliseront ces capacités pour faire avancer rapidement les acheteurs dans le processus de décision, peu importent les circonstances.

\section{Références}

Benchmark Group (2007), Commerce électronique : performances comparées des sites, chiffres clés, $\mathrm{http} / / / \mathrm{www}$. benchmark.fr/catalogue/publication/67/commerce-electronique-performancescomparees-des-sites-chiffres-cles.shtml.

Benchmark Group (2009), Processus de commande sur les sites marchands : les meilleures pratiques, http://www.benchmark.fr/catalogue/publication/90/etude-processus-de-commande-sur-les-sitesmarchands-les-meilleures-pratiques.shtml\#sommaire.

Monster, R., Pettit, R., Cowling, T. (2002), Market Research in the Internet Age : Leveraging the Internet for Market Measurement and Consumer Insight, John Wiley and Sons, 306 p. 